\title{
Design of Mach-Zehnder Interferometer and Ring Resonator for Biochemical Sensing
}

\author{
Dedi IRAWAN ${ }^{1,2^{*}}$, Toto SAKTIOTO ${ }^{1,3}$, Jalil ALI $^{1}$, and Preecha YUPAPIN ${ }^{4}$ \\ ${ }^{1}$ Advanced Photonics Science Institute (APSI), Faculty of Science, Universiti Teknologi Malaysia, Johor 81310, Malaysia \\ ${ }^{2}$ Dept. of Industrial Engineering, Faculty of Science and Technology, UIN Suska Pekanbaru, Indonesia \\ ${ }^{3}$ Dept. of Physics, Math and Science Faculty, University of Riau, Pekanbaru, Indonesia \\ ${ }^{4}$ Dept. of Physics, King Mongkut's of Technology Ladkrabang and Quantum Life Institute, Nonthaburi, Thailand \\ ${ }^{*}$ Corresponding author: Dedi IRAWAN_ E-mail: dedi.dawan@yahoo.com
}

\begin{abstract}
We proposed a compact design of an optical biochemical sensor based on the Mach-Zehnder interferometer (MZI), which was coupled by a ring resonator (RR) as a sensing tool. The sensor sensitivity has been determined by power difference at the output ports. The sensor enhancement has been optimized by numerically evaluating the geometrical parameters of the MZI and RR. A great sensor sensitivity depicted by Fano resonance characteristic has been demonstrated as a function of the round trip phase in the range of $4 \times 10^{-4}-4 \times 10^{-4}$, which was changed by the presence of the sample solution in the sensing area. This optimum sensitivity has been obtained for the values of two coupling coefficients of the MZI $\kappa_{1}=\kappa_{2}=0.5 / \mathrm{mm}$ and the coupling coefficient between the MZI arm and RR $\kappa_{R}=0.5 / \mathrm{mm}$. Furthermore, a good profile of sensitivity exchange has been exhibited by inducing the direct current voltage to the coupling region of $\kappa_{R}$. Finally, the output power transmission of the ring-coupled arm was depicted as a function of tunable $\kappa_{R}$.
\end{abstract}

Keywords: Mach-Zehnder interferometer, ring resonator, biochemical sensor, tunable sensitivity, Fano resonance

Citation: Dedi IRAWAN, Toto SAKTIOTO, Jalil ALI, and Preecha YUPAPIN, "Design of Mach-Zehnder Interferometer and Ring Resonator for Biochemical Sensing," Photonic Sensors, 2015, 5(1): 12-18.

\section{Introduction}

The development of the optical waveguide for the biosensing application has greatly expanded. Since it works based on real-time measurement, which has no electrical interference, it promises a wide range of the research application such as for bio medic, health care, pharmaceutical, security, and military defence. In the past two decades, many researchers have focused on the experimental and theoretical studies of the optical waveguide application for biochemical sensing. The gold properties of the optical waveguide have been shown as a function of effective refractive index change due to evanescence and fluorescence fields when it interacts with the sample [1,2].

Many optical sensors were developed based on the fiber optic sensor (FOS), surface plasmon resonance (SPR), photonic wire (PW), Mach-Zehnder interferometer (MZI), and ring resonator (RR) [3]. A chemical sensing was designed by coupled slot silicon on insulator (SOI) waveguides. The sensor performance was numerically calculated based on the principle of the

Received: 12 June 2014 / Revised version: 15 July 2014

(C) The Author(s) 2014. This article is published with open access at Springerlink.com

DOI: $10.1007 / \mathrm{s} 13320-014-0200-5$

Article type: Regular 
directional fiber coupler in which one of its slots was attached by a sample solution. The frequency responding to the refractive index change was exhibited for quasi-TE and quasi-TM modes [4]. However, the use of both the MZI and RR for biosensing becomes an interesting field to be studied based on its easy fabrication and high sensitivity.

A biochemical sensor based on asymmetrical resonance was investigated for a great potential application of highly sensitive sensors due to $Q$ factor by utilizing a polymer micro RR [5]. This system showed the sensor sensitivity enhancement as a function of the Fano resonance line shape which was varied as the concentration of the glucose solution changed. Separately, the use of the MZI for the high sensitivity sensor was proposed based on the bent and tapered fiber [6]. A good sensitivity refractive index sensing was purposed by an electric-arc induced MZI in a thinned cladding fiber [7]. The sensitivity was produced in the rage of about $580 \mathrm{~nm} /$ refractive index unit (RIU) which was 20 times greater than that of a taper MZI. A sensor system based on dual MZIs, which were designed using single mode fiber (SMF), was also investigated for a liquid analyte. The sensor enhancement was proposed by integrating $\mathrm{Ta}_{2} \mathrm{O}_{5}$ with the reference structure.

Recent study has investigated a biochemical sensing design by attaching a ring in the arm of the MZI. They also optimized numerically the sensing sensitivity by determining MZI and RR parameters such as the coupling coefficient, ring's radius, and phase reference. The high sensitivity MZI coupled by two rings was also proposed. This study exhibited the intensity change of the Fano resonance which was detected at the fixed wavelength. In some cases, various biochemical samples change highly the characteristics of the sensing waveguide or vice versa, so a tunable sensitivity sensor is required to enhance the sensor performance [8].

Many potential applications can be covered by a single optical sensor device if it provides an adjustable sensitivity system. A biophotonic waveguide was purposed as a tunable and sensitive sensor system based on photonic-bandgap microcavities. The refractive index was successfully changed by inducing the waveguide with two electrodes with positive and negative polarities. However, this sensor system requires the high cost fabrication and advanced technique [9].

In this paper, we propose a simple design of the unbalanced Mach-Zehnder interferometer attached by the ring resonator with an adjustable coupling between the MZI and RR based on the sensor configuration. The geometrical parameters of the MZIRR design such as the coupling coefficients of the MZI and ring, ring's radius, phase reference, and the round-trip phase were numerically investigated. The sensing sensitivity was determined based on power output difference at the output ports of the MZI. This method is a powerful technique to determine the sensitivity since the power output difference changes greater than the power output at both output ports. Furthermore, a sensitivity change as a tunable sensitivity was then calculated as a function of the applied voltage based on Pockel's effect model. The power transmission was then shown as a function of the ring's coupling coefficient. Finally, we showed the sensing performance by exhibiting the optical characteristics of the Fano resonance.

\section{Design of MZIRR}

A sensor design shown in Fig. 1 was proposed as an unbalanced ring coupled to one arm of the MZI. An input power of the laser diode $P_{\text {in }}=1 \mathrm{~mW}$ is launched into the input port which is then split by a 3 -dB coupler $\kappa_{1}$ into two arms of the MZI. The first signal travels along the upper arm, and part of light is split by $\kappa_{R}$ and enters the ring as a sensing area with a radius $R$.

After propagating inside the ring, this signal will be split, and part of it travels along the arm and then is coupled by the fiber coupler 2 before it is released 
to the output port $1 P_{\text {out } 1}$. However, the second signal travels in the lower arm as a reference signal and is coupled by the second fiber coupler before it is released to the output port $2 P_{\text {out } 2}$.

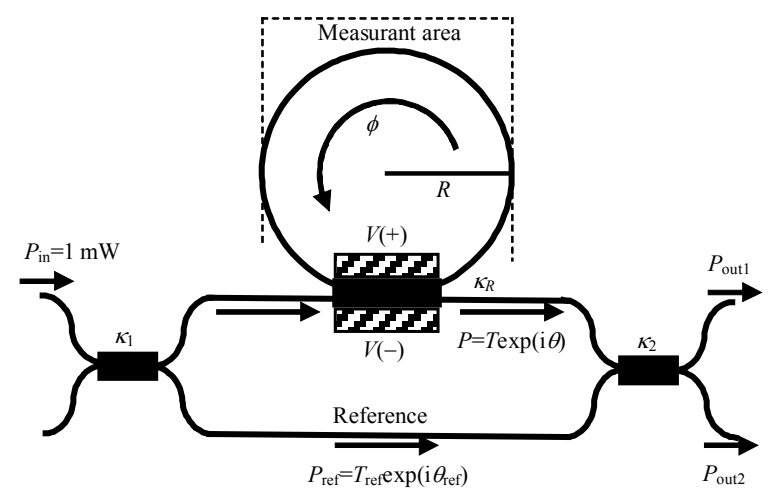

Fig. 1 Sketch of the MZIRR based on the biosensing configuration.

For a single MZI without a ring resonator, the output power is determined by the coupling coefficient of two couplers $\kappa_{1}$ and $\kappa_{2}$ which is expressed in term of the matrix transform as follows [10]:

$$
\left[\begin{array}{c}
E_{\text {out1 }} \\
E_{\text {out } 2}
\end{array}\right]=\left[\begin{array}{cc}
-s^{2}+c_{1}^{2} z^{-1} & -\mathrm{i} c s\left(1+z^{-1}\right) \\
-\mathrm{i} c s\left(1+z^{-1}\right) & c_{2}^{2}-s^{2} z^{-1}
\end{array}\right]\left[\begin{array}{c}
E_{\text {in } 1} \\
E_{\text {in } 2}
\end{array}\right]
$$

where $c_{1}=\sqrt{1-\kappa_{1}}$ and $c_{2}=\sqrt{1-\kappa_{2}}$ are the constants of the coupling coefficient of the MZI, and $\mathrm{i} s=-\mathrm{i} \sin (\theta)=-\mathrm{i} \sqrt{\kappa}$.

However, the presence of a ring resonator at MZI's arms causes the signals split in two parts, one passing through inside the arm and the other traveling inside the ring. The power ratio is contributed by a coupling coefficient between the ring and the upper arm of the MZI $\kappa_{R}$. For example, if one ring is coupled at one of MZI's arm, the output power is given as follows [8]:

$$
\begin{aligned}
& E_{\text {out } 1}=\left[c^{2} \kappa_{R}-s^{2} \exp \left(-\mathrm{i} \theta_{\text {ref }}\right)\right]- \\
& \left\{c^{2} \exp (-\mathrm{i} \phi)-s^{2} c_{R} \exp \left[-\mathrm{i}\left(\phi+\theta_{\text {ref }}\right)\right]\right\} E_{\text {in }}
\end{aligned}
$$

where $c_{R}=\sqrt{1-\kappa_{R}}$ are constants of the coupling coefficient between the MZI and ring resonator.

When the signals circulate inside the ring, the round-trip phase will be induced by the attenuation and the ring's radius $R$. Although a propagation constant of the fiber is $\beta=2 \pi n_{\text {eff }} / \lambda$, the round-trip phase can be expressed as follows:

$$
\phi=m R \beta
$$

where $m$ is an integer $(0,1,2, \cdots)$, and $n_{\text {eff }}$ denotes the effective refractive index.

\section{Tunable sensitivity of MZIRR}

The sensor sensitivity of the MZIRR can be determined in several ways, by determining the frequency or wavelength difference at the output port 1 then compared with the reference signal. In the conventional MZI based sensor, the sensitivity is determined by a change in signal properties of the single output signal only. It can be seen that a change in power difference is faster than the single output change. In this work, we proposed an idea to enhance the sensitivity by evaluating the change in power difference at two output powers of the MZI. Firstly, let us define power difference $P_{\text {diff }}=P_{\text {out2 }}-P_{\text {out1 } 1}$, where $\quad P_{\text {out1 }}=\left|E_{\text {out } 1}\right| \quad$ and $P_{\text {out } 2}=\left|E_{\text {out } 2}\right|$.

By introducing the sensor sensitivity which is changed by the presence of the solution $\Omega$, we can then express it as follows:

$$
S=\frac{P_{\text {diff }}}{d \Omega} \Rightarrow S=\frac{P_{\text {diff }}}{d \phi} \cdot \frac{d \phi}{d \Omega} .
$$

Equation (3) describes that the sensitivity is determined by power output difference due to the change in the round trip phase $\phi$ which is induced by the sensing $\Omega$. However, it is not easy to determine $P_{\text {diff }} / d \phi$. The difference of power outputs between two output ports is mathematically expressed as a function of the coupling coefficient of $\kappa_{1}$ and $\kappa_{2}$, round-trip phase $(\phi)$, and reference phase $\left(\theta_{\text {ref }}\right)$.

$$
\begin{aligned}
\frac{d P_{\text {diff }}}{d \phi}= & \left\{-\left[\left(4 A \cos \left(\theta_{\text {ref }}-\theta(\phi)\right)+2 B T(\phi)\right]\right.\right. \\
& \left.\left.T^{\prime}(\phi)-4 A \sin \bigoplus_{\text {ref }}-\theta(\phi)\right) T(\phi) \theta^{\prime}(\phi)\right\}\left|E_{\text {in }}\right|
\end{aligned}
$$

where $A=\sqrt{\kappa_{1} \kappa_{2} c_{1} c_{2}}$ and $B=\kappa_{1}+\kappa_{2}-2 \kappa_{1} \kappa_{2}-1$. When the input signal travels inside the ring, the transmitted amplitude and phase are $T(\phi)=\left|E_{\text {out }}\right|$ and $\theta(\phi)=\arg \left(E_{\text {out }}\right)$, respectively, then $T^{\prime}$ and 
$\theta^{\prime}$ are denoted as their first derivatives.

To adjust the sensor sensitivity, it can reasonably be carried out by changing the coupling coefficient between the arm and ring. Although (5) is a function of the coupling coefficient of the MZI, we need to introduce the coupling coefficient between the arm and ring into our calculation. If the input power entering the ring is $E_{\text {in }}$ and by determining that the power loss along the ring is a function of ring's radius $R$ and attenuation constant $\alpha$, the electric field output of the ring $E_{\text {outR }}$, coupled at the arm MZI, is expressed as the function of ring's coupling coefficient $\kappa_{R}$ and phase of the round trip signal $\phi$ as follows:

$$
E_{\text {outR }}=\frac{\exp (\mathrm{i} \phi-\pi R a)-c_{R}}{c_{R} \exp (\mathrm{i} \phi-\pi R a)-1} E_{\text {in }} .
$$

Since the optical loss in the arm of the MZI is ignored in our calculation, all optical power will be normalized, and the resonance inside the ring exhibits that $c=1-\kappa_{R} / 2$. Then, the coupling coefficient of the ring will be also normalized as $\kappa_{\text {normalized }}=\kappa_{R} / 2 \pi R a$ with the normalized phase round trip $\phi_{\text {normalized }}=\phi / 2 \pi R a$. Now (6) can be simplified as

$$
E_{\text {outR }}=\frac{1-\kappa_{\text {normalized }}-2 \mathrm{i} \phi_{\text {normalized }}}{1+\kappa_{\text {normalized }}-2 \mathrm{i} \phi_{\text {normalized }}} E_{\text {in }} .
$$

Now, we propose to adjust the coupling coefficient of the ring by applying the direct current voltage to its coupling region. For identical fiber of the MZI and ring, the coupling coefficient is empirically expressed as a function of the separation between MZI' arm and ring $d$, and fractional refractive indices $\delta=\frac{n_{\mathrm{co}}^{2}-n_{\mathrm{cl}}^{2}}{n_{\mathrm{co}}^{2}}=\Delta \beta / 2$ as follows [11]:

$$
\kappa=\frac{\pi}{2} \frac{\sqrt{\delta}}{R} \mathrm{e}^{\left[-A+B \partial+C \partial^{2}\right]}
$$

where

$$
\begin{aligned}
& A=5.2789-(3.663 V)+\left(0.3841 V^{2}\right) \\
& B=-0.7769-(1.2252 V)+\left(0.0152 V^{2}\right) \\
& C=-0.0175-(0.0064 V)+\left(0.0009 V^{2}\right) \\
& \partial=d / \delta .
\end{aligned}
$$

The effect of the applied direct current voltage in the coupling region is described by Pockel's effect as follows [12]:

$$
\Delta n(\lambda, y, V)=-\frac{n_{\mathrm{co}}^{3}}{2} R E(x, y)
$$

where $\Delta n=n_{\mathrm{co}}-n_{\mathrm{cl}}$. Since the signal is applied perpendicular to the cross section of the coupling length with the distance of two electrodes $x$ and electric field $E=V_{\mathrm{dc}} / x$, (9) can be simplified as

$$
n_{\mathrm{co}}^{3}=\left[n_{\text {co(initial) }}(2-2 \sqrt{1-\delta}-\delta)^{1 / 2}\right] \frac{2 x}{R V_{\mathrm{dc}}} .
$$

Equations (8) and (10) show that applying the direct current (DC) voltage to the coupling coefficient will induce the coupling region between the MZI's arm and ring.

\section{Results and discussion}

Power exchanges between output powers $P_{1}$ and $P_{2}$ lead to the change in the power difference ( $\left.P_{\text {diff }}=P_{1}-P_{2}\right)$ which determines the sensor sensitivity. We numerically evaluate the coupling coefficient of the MZI, $\kappa_{1}, \kappa_{2}, \theta_{\text {ref }}$, and $P_{\text {ref }}$. In our calculation, we confine a range of the round trip phase change from $-4 \times 10^{-4}$ to $4 \times 10^{-4}$. The optimum design is obtained when the coupling coefficient and phase of the ring are $\kappa_{1}=\kappa_{1}=0.5$ and $\theta_{\text {ref }}= \pm \pi / 2$, respectively.

Based on Fig. 1, it is reasonable that the maximum sensitivity can be reached for $P_{\text {ref }}=1$. Since the coupling coefficient of the MZI divides the input source signal with the splitting ratio of 50\%:50\%, the output powers detected at both output ports are symmetrically changed [see Fig. 2(a)] as the Fano-resonance profile. Otherwise, a minimum sensitivity is numerically obtained for a value of phase reference $\theta_{\text {ref }} \approx 0$ and for an input power amplitude $P_{\text {ref }}$.

The slope of the Fano resonance is significantly affected by the coupling coefficient between the arm and ring resonator $\kappa_{R}$ and the phase signals traveling in the non-resonance arms. The optimized sensitivity can be described by the slope of power 
difference in Fig. 2(b) which is greater than single output power.

Figure 2(b) depicts the normalized power difference as a function of the round trip phase which is changed due to sample-ring interaction. Normally in the biochemical application, the significant change occurs in the range of the round trip phase from $-10^{-5}$ to $10^{-5}$. By calculating the change in the refractive index using (3), it yields the range exchange about $10^{-7}$ RIU (refractive index unit).

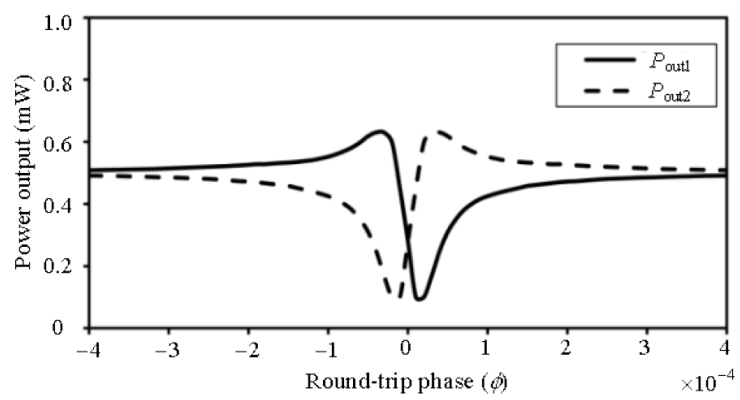

(a)

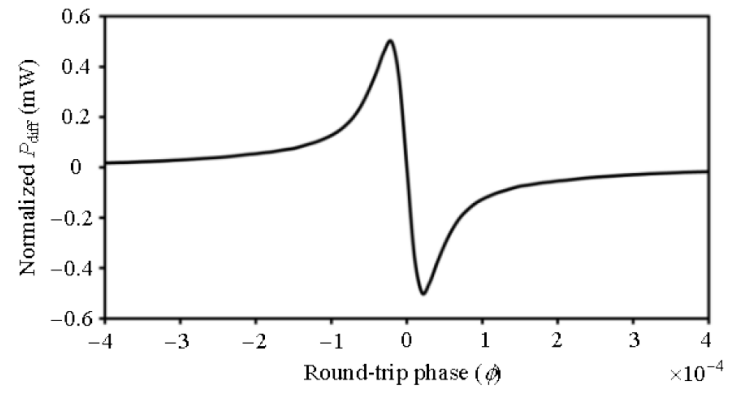

(b)

Fig. 2 Output power characteristics of the MZIRR based sensor changed by the round trip phase: (a) Fano resonance provided by outputs $P_{1}$ and $P_{2}$ and (b) normalized power difference.

However, the characteristics of the Fano resonance will also be induced by a factor of $2 \pi R \alpha$ according to (5). This means that to enhance the gradient of the Fano resonance, we need to reduce the ring radius and attenuation. In fact, the method cannot be applied to the smaller ring radius since the Kerr effect will take place in our calculation. Only minimizing the attenuation due to the bending loss is a better way to optimize the system.
Equation (6) shows that the output power released by the ring much affects its coupling coefficient $\kappa_{R}$, and this becomes a good approximation to adjusting the power output due to the change in the sensitivity range. Here, we induce the coupling region of $\kappa_{R}$ by applying the DC voltage from $0 \mathrm{~V}$ to $10 \mathrm{~V}$. The applied DC voltage which is perpendicular to the fiber length induces the permeability of the fiber characteristics. This disturbance will be a polarization function of the electric field passing through the fibers. As a result, it affects the fiber refractive index as described by Pockel's effect model given by (9).

Figure 3 is obtained by calculating the refractive index based on an increment of the DC voltage from $0 \mathrm{~V}$ to $25 \mathrm{~V}$. The significant change occurs below than $10 \mathrm{~V}$ only. For a higher voltage such as $V_{\mathrm{dc}}>>25$, more breakdowns will take place and produce more coupling losses.

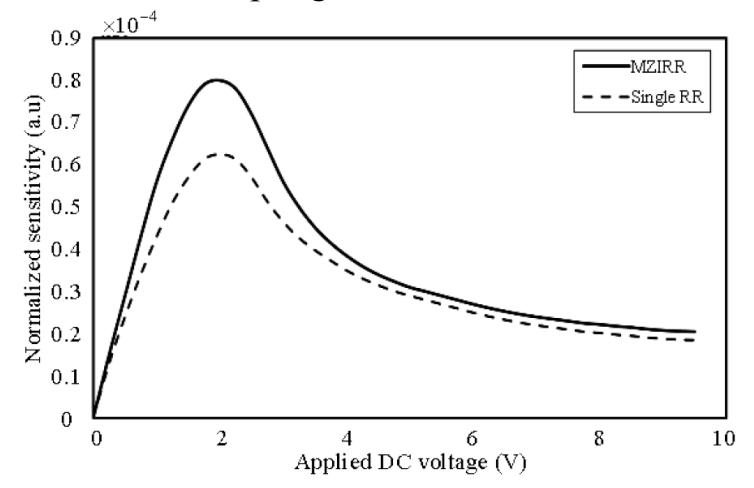

(a)

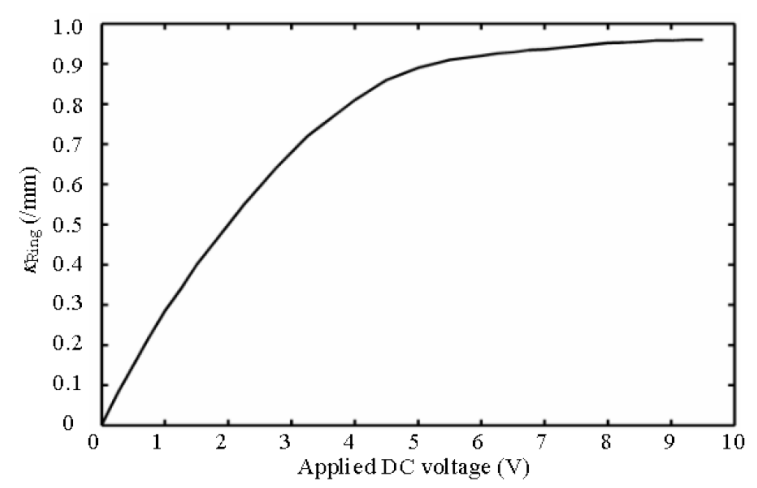

(b)

Fig. 3 Sensing sensitivity characteristics of the MZIRR: (a) sensitivity profile of the MZIRR controlled by the applied DC voltage and (b) coupling coefficient enhancement of the ring resonator. 
It can be seen that the sensitivity increases sharply until it reaches a maximum for a value of the $\mathrm{DC}$ voltage equal to $2 \mathrm{~V}$. We note this condition obtained when the coupling coefficient $\kappa_{R}$ is equal to 0.5 [see Fig. 3(b)]. In this condition, the input signal is split symmetrically into the ring and through the upper arm. By increasing the applied DC voltage, it leads to a decrease in the sensitivity exponentially until it remains constant for $\kappa_{R}>>1$. The proposed sensor design based on the MZIRR also exhibits the higher sensitivity than a conventional single RR with a good agreement as shown by the dash line.

A range of the applied DC voltage to control the sensitivity is not actually an exact number which in our case varies from $0 \mathrm{~V}$ to $10 \mathrm{~V}$. This range significantly depends on the separation distance between two electrodes in the coupling region: the longer the separation distance is, the higher the DC voltage is required to change the sensor sensitivity. In our consideration, two electrodes are positioned to the coupling region to reduce the electrode-air-fiber interface.

Figure 4 exhibits the power transmission of the upper arm after the signal is released by the ring resonator as a function of applied normalization of the ring coupling coefficient. The power at the upper arm is reduced until the minimum at the ring coupling of $\kappa_{R}=1 / \mathrm{mm}$ which means all initial input power enters and travels inside the ring. However, Fig. 4 is generated by assuming all input power is in the upper arm. More increment of the ring coupling $\kappa_{R}$ causes an increase in the power transmission and remains constant for $\kappa_{R}>16$.

Finally, the optimum sensor design could be achieved by determining a set of MZI parameters including the coupling coefficient between MZI's arm and ring resonator. However, the optical loss in the ring resonator still takes place since we cannot increase the ring radius due to the attenuation coefficient. For the fabrication process, we suppose to minimize the coupling loss at MZI coupling because it also affects the sensor sensitivity.

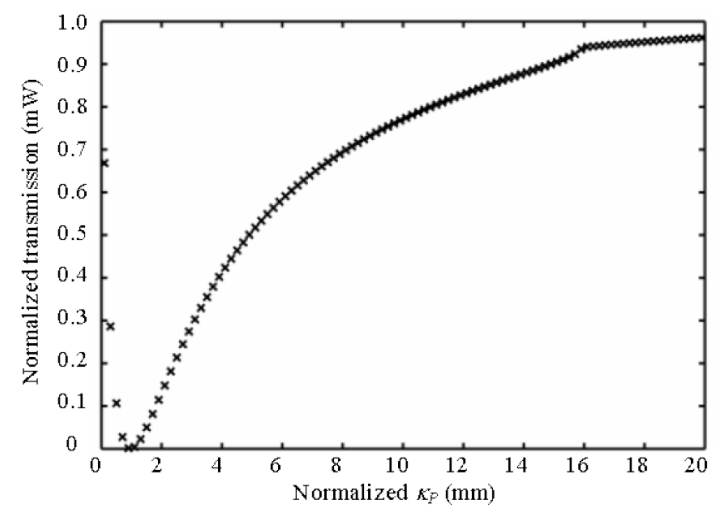

Fig. 4 Power transmission changed by the normalized coupling coefficient.

In the ring coupling region, the use of the DC voltage to change the coupling region also contributes the optical loss which is not considered in our calculation. This method can be replaced by the mechanical process for easier coupling control, for example by changing mechanically the separation distance between the arm and ring resonator.

\section{Conclusions}

A tunable sensitivity of the MZIRR for bio-sensing is successfully optimized by determining the sensitivity based on power difference at the output ports of the MZI and applying a direct current voltage in the range of $0 \mathrm{~V}$ $-10 \mathrm{~V}$. A good agreement between the sensitivity plot and applied voltage is obtained by setting the coupling coefficient of the MZI equal to $0.5 / \mathrm{mm}$. But the ring coupling is adjustable under the control of the DC voltage. A Fano resonance characteristic of output power of the MZI is exhibited as sensor enhancement for the reference phase and amplitude $\theta_{\text {reff }}= \pm \pi$ and $P_{\text {reff }}=1$, respectively. The effect of the applied voltage on the sensitivity is due to the change in the fiber permeability that leads to the change in the refractive index.

\section{Acknowledgment}

We would like to thank the Institute of Advanced Photonic Science (APSI) Faculty of 
Science, Universiti Teknologi Malaysia, Faculty of Science and Technology UIN Suska Riau, and University of Riau Indonesia for great support in this research.

Open Access This article is distributed under the terms of the Creative Commons Attribution License which permits any use, distribution, and reproduction in any medium, provided the original author(s) and source are credited.

\section{References}

[1] M. Janotta and B. Mizaikoff, "Recent developments in liquid phase mid-infrared sensor technology," in Proc. SPIE, vol. 4616, pp. 1-8, 2002.

[2] M. R. Safadi, A. Scott, J. Thakur, and G. Auner, "Fabrication of a waveguide for biomedical applications using novel micromachining techniques," in Proc. SPIE, vol. 588, pp. 205-213, 2004.

[3] X. Fan, I. M. White, S. I. Shopova, H. Zhu, J. D. Suter, and Y. Sun, "Sensitive optical biosensors for unlabeled targets: a review," Analytica Chimica Acta, 2008, 620 (1-2): 8-26.

[4] V. M. N. Passaro, F. Dell'Olio, C. Ciminelli, and M. N. Armenise, "Efficient chemical sensing by coupled slot SOI waveguides," Sensors (Basel), 2009, 9(2): 1012-1032.

[5] C. Y. Chao and L. J. Guo, "Biochemical sensors based on polymer microrings with sharp asymmetrical resonance," Applied Physics Letters, 2003, 83(8): $1527-1529$.

[6] J. Chen, J. Zhou, and Z. Jia, "High-sensitivity displacement sensor based on a bent fiber MachZehnder interferometer," IEEE Photonics Technology Letters, 2013, 25(23): 2354-2357.

[7]B. Li, L. Jiang, S. Wang, Q. C. M. Wang, and J. Yang, "A new Mach-Zehnder interferometer in a thinned-cladding fiber fabricated by electric arc for high sensitivity refractive index sensing," Optics and Lasers in Engineering, 2012, 50(6): 829-832.

[8] J. Zhang, Y. Zhang, Q. Song, H. Tian, X. Zhang, $\mathrm{H}$. Wu, et al., "A double-ring Mach-Zehnder interferometer sensor with high sensitivity," Journal of Physics D: Applied Physics, 2012, 45(25): 1-8.

[9] C. E. Png, S. T. Lim, E. P. Li, and G. T. Reed, "Tunable and sensitive biophotonic waveguides based on photonic-bandgap microcavities," IEEE Transactions on Nanotechnology, 2006, 5(5): 478-484.

[10] P. Yupapin, C. Teeka, and J. Ali, Nanoscale nonlinear Panda ring resonator. London: CRC Press, 2012.

[11] A. Ghatak and K. Thyagarajan, An introduction to fiber optics. Cambridge: Cambridge University Press, 1998.

[12] D. Irawan, Saktioto, J. Ali, and Defrianto, "Breakdown voltage effect on coupling ratio fusion fiber coupling," Physics Procedia, 2011, 19: 477-481. 